\title{
ON WHITEHEAD'S ALGORITHM
}

\author{
BY S. M. GERSTEN ${ }^{1}$
}

\begin{abstract}
One can decide effectively when two finitely generated subgroups of a finitely generated free group $F$ are equivalent under an automorphism of $F$. The subgroup of automorphisms of $F$ mapping a given finitely generated subgroup $S$ of $F$ into a conjugate of $S$ is finitely presented.
\end{abstract}

In two famous articles $[9,10]$ which appeared in 1936, J. H. C. Whitehead, using the theory of three-dimensional handlebodies, proved that one can effectively decide when two $n$-tuples of cyclic words of a finitely generated free group $F$ are equivalent by an automorphism of $F$. The proof of this result has been simplified successively $[7,3]$ and the result itself has been immensely influential. Whitehead himself poses the problem of generalizing his theorem $[10$, p. 800$]$; namely he raises the question of deciding when two finitely generated subgroups of $F$ are equivalent by an automorphism of $F$.

In $1974 \mathrm{McCool}$ [6] deduced a profound consequence of Whitehead's theorem, proving that the stabilizer, in the automorphism group of $F$, of an $n$ tuple of cyclic words is finitely presented. Using graph-theoretic techniques we developed in [1] (the results of which were announced in [2]), we have succeeded both in settling Whitehead's question and in generalizing McCool's results.

Let $A$ denote the automorphism group of $F$, and let $S$ denote the set of conjugacy classes of finitely generated subgroups of $F$ with its natural $A$ action. Let $S^{n}$ denote the cartesian product of $n$ copies of $S$ with diagonal $A$ action.

THEOREM W. There is an effective procedure for determining when two elements of $S^{n}$ are in the same orbit of the A-action.

TheOREM M. The stabilizer in $A$ of an element of $S^{n}$ is finitely presented, and a finite presentation can be effectively determined.

In this note we indicate briefly the ideas that go into the proofs of Theorems $\mathrm{W}$ and $\mathrm{M}$. Full details will appear elsewhere.

We use the theory of graphs defined in [2]. A graph $X$ is a nonempty set with involution, denoted $x \mapsto \bar{x}$, together with a retraction $\iota: X \rightarrow V(X)$ of $X$ onto the fixed point set $V(X)$ of the involution. Morphisms of graphs preserve the involution and the retraction. The set $V(X)$ is called the set of vertices of

Received by the editors September 30, 1983.

1980 Mathematics Subject Classification. Primary 20F34.

Key words and phrases. Free group, automorphism, graph, finitely presented.

${ }^{1}$ Partially supported by NSF Grant MSC 83-01798 
$X$ and $E(X)=X-V(X)$ is called the set of edges. A morphism $f: X \rightarrow X^{\prime}$ of graphs is called an immersion [8] if for each $v \in V(X)$ the induced map $f_{v}: \operatorname{Star}_{X}(v) \rightarrow \operatorname{Star}_{X^{\prime}}(f(v))$ is injective; here $\operatorname{Star}_{X}(v)=\{x \in X \mid \iota x=v\}$. A graph $X$ is called a core graph if it has no end vertices, where $v \in V(X)$ is called an end vertex if there exists precisely one edge $e$ with $\iota e=v$. The graphs considered in this note (except for coverings in parenthetical remarks) are all finite.

Suppose now that $j: X \rightarrow Y$ is an immersion of the core graph $X$ in the 1vertex graph $Y$ (i.e. $\# V(Y)=1$ ). Define the complexity of $j$, or of $X$ by abuse of notation, to be $\# V(X)$ and denote it by $c(X)$. The crucial algebraic result below will enable us to compute the effect of a Whitehead automorphism of $\pi_{1}(Y)[4, \mathrm{p} .31]$ on the complexity $c(X)$. (That $A=\operatorname{Aut} \pi_{1}(Y)$ acts on immersions $X \stackrel{j}{\rightarrow} Y$ may be seen as follows. If $X$ is connected, and $v \in V(X)$, then $j$ injects $\pi_{1}(X, v)$ into $\pi_{1}(Y)$ to determine a conjugacy class of subgroups of $\pi_{1}(Y)$. If $\alpha \in A$, represent the subgroup $\alpha\left(j\left(\pi_{1}(X, v)\right)\right)$ of $\pi_{1}(Y)$ as a covering of $Y$ and take a core of the covering to get the desired immersion $\alpha(X) \stackrel{\alpha(j)}{\rightarrow} Y$.)

If $A, B \subseteq E(Y)$ and $v \in V(X)$, define $(A \cdot B)_{v}$ to be 1 if there exists a reduced path $e e^{\prime}$ in $X$ (so $e, e^{\prime} \in E(X)$ ) with $\iota \bar{e}=\iota e^{\prime}=v, j e \in A$, and $j\left(\overline{e^{\prime}}\right) \in B$, and let $(A \cdot B)_{v}$ be 0 otherwise. Set $A \cdot B=\sum_{v \in V(X)}(A \cdot B)_{v}$. Thus $A \cdot B$ is the number of vertices $v$ of $X$ for which a reduced path $e e^{\prime}$ exists in $X$ with $j(e) \in A$, $j\left(\overline{e^{\prime}}\right) \in B$, and $\iota(\bar{e})=\iota\left(e^{\prime}\right)=v$.

Proposition 1. If $\alpha=(A, a)$ is a Whitehead automorphism of $\pi_{1}(Y)(A \subset$ $E(Y), a \in A, \bar{a} \notin A)$ and $X \stackrel{j}{\rightarrow} Y$ is an immersion of the core graph $X$ in the one vertex graph $Y$, then $c(\alpha(X))-c(X)=A \cdot A^{\prime}-\{a\} \cdot E(Y)$. Here $A^{\prime}=E(Y)-A$.

This result reduces to Proposition 4.16, p. 31 of [4] in the special case when $X$ is the graph whose geometric realization is a subdivision of the circle. The formal properties of the pairing $A \cdot B$ are:

(1) $A \cdot B=B \cdot A \geq 0$;

(2) $\{a\} \cdot\{a\}=0$ if $a \in E(Y)$,

(3) $\{a\} \cdot E(Y)=\#\left\{v \in V(X) \mid \exists e \in \operatorname{Star}_{X}(v)\right.$ with $\left.f(e)=a\right\}=\{\bar{a}\} \cdot E(Y)$, if $a \in E(Y)$.

The pairing $A \cdot B$ is not bilinear over disjoint unions, unlike the special case considered in $[4$, p. 31]. However a weaker result holds.

Proposition 2. For any subsets $A, B$ of $E(Y)$ one has

$$
A \cdot A^{\prime}+B \cdot B^{\prime} \geq(A \cap B) \cdot(A \cap B)^{\prime}+\left(A^{\prime} \cap B^{\prime}\right) \cdot\left(A^{\prime} \cap B^{\prime}\right)^{\prime} .
$$

In fact the analogous inequality holds locally at each vertex of $X$.

Proposition 3. Let $A$ and $B$ be subsets of $E(Y)$ with $A \cap B=\varnothing, a \in A$, $\bar{a} \notin A, b \in B, \bar{b} \notin B$, and $\bar{a} \notin B$. Let $\sigma=(A, a)$ and $\tau=(B, b)$ be Whitehead automorphisms of $\pi_{1}(Y)$. Then for any immersion $j: X \rightarrow Y$ of the core graph $X$ into the 1-vertex graph $Y$, one has

$$
c(\tau \sigma(X))-c(\sigma X)=c(\tau X)-c(X) \text {. }
$$


Using Propositions 1-3 and following the plan of the argument of Lemma 4.18 of [4], one proves

TheOREM 1. Suppose $j: X \rightarrow Y$ is an immersion, where $X$ is a core graph and $Y$ is a 1-vertex graph. Let $\sigma$ and $\tau$ be Whitehead automorphisms of $\pi_{1}(Y)$ such that $c(\sigma(X)) \leq c(X)$ and $c(\tau(X)) \leq c(X)$, where at least one inequality is strict. Then using only McCool's relations $\mathrm{R} 1-\mathrm{R} 7$ [5] one has $\tau \sigma^{-1}=$ $\sigma_{m} \cdots \sigma_{2} \sigma_{1}$, where $\sigma_{i}$ are Whitehead automorphisms and where $c\left(\sigma_{i} \cdots \sigma_{1} \sigma(X)\right)$ $<c(X)$ for $1 \leq i<m$.

Suppose now that $S$ is a conjugacy class of finitely generated subgroups of $\pi_{1}(Y)$. Then $S$ determines (by taking a covering of $Y$ and taking a core of the cover) an immersion $j: X \rightarrow Y$ of a finite core graph $X$ in $Y$ such that $j\left(\pi_{1}(X, v)\right)$ is in the conjugacy class $S$; the graph $X$ is unique up to isomorphism, so we may define the complexity

$$
c(S)=c(X)=\# V(X) \text {. }
$$

Observe that in the special case where $S$ is represented by the cyclic group $\langle w\rangle, c(S)$ is just the length of a cyclically reduced word conjugate to $w$. Observe also that if some representative of $S$ has finite index $n$ in $\pi_{1}(Y)$ (whence all representatives have index $n$ ) then $c(S)=n$, since the immersion corresponding to $S$ is an $n$-fold covering space of $Y$ in this case.

COROLlaRY 1. Let $F$ be a finitely generately free group with given free basis $O$ and let $S=\left(S_{1}, S_{2}, \ldots, S_{n}\right)$ be an $n$-tuple of conjugacy classes of finitely generated subgroups of $F$. Let $c(S)=\sum_{i=1}^{n} c\left(S_{i}\right)$. Suppose that $\sigma$ and $\tau$ are Whitehead automorphisms of $F$ such that $c(\sigma(S)) \leq c(S)$ and $c(\tau(S)) \leq c(S)$ with at least one inequality strict. Then using only $M c$ Cool's relations $\mathrm{R} 1-\mathrm{R} 7$ one has $\tau \sigma^{-1}=\sigma_{m} \cdots \sigma_{2} \sigma_{1}$, where $\sigma_{i}$ are Whitehead automorphisms and where $c\left(\sigma_{i} \cdots \sigma_{1} \sigma(S)\right)<c(S)$ for $1 \leq i<m$.

An immediate consequence of Corollary 1 is

COROLlaRY 2. If $c(S)$ can be reduced by some automorphism of $F$, then it can be reduced by a Whitehead automorphism.

Theorem W follows from Corollary 2 by the method of proof of Proposition 4.19 of $[4]$.

We remark that Theorem $\mathrm{M}$ follows from Corollary 1 by arguments mimicking McCool's [6].

EXAMPLE. Suppose $S$ is a finitely generated subgroup of $F$ whose conjugacy class has complexity 1 . Then using Stallings' form of Marshall Hall's theorem [8] it follows that $S$ is a free factor of $F$. Whitehead found another algorithm to detect when $S$ is a free factor of $F$, based on the existence of a cut vertex in the (based) star graph of a basis for $S$ [9]. We have also given a direct proof of this result using our graph techniques, avoiding any use of handlebodies.

REMARK. The novel feature of our work is our definition of the complexity $c(S)$ of an $n$-tuple $S$ of conjugacy classes of finitely generated subgroups of a free group. Whitehead's own example [10, p. 800], indicating 
the difficulty of his problem of deciding when two $f g$ subgroups of the free group $F$ were equivalent, when reexamined in this light, shows that he was working with the wrong notion of complexity of a subgroup (he uses the sum of lengths of the elements of a given free basis for a subgroup). It is our complexity, defined in terms of the core of a covering space, which satisfies the correct transformation formula under Whitehead automorphisms, so that Whitehead's own arguments will work. Whitehead's examples [10, p. 800], $S=\left\langle(a \bar{b})^{2} \bar{b}^{2}(a \bar{b})^{2} a^{3}, \bar{a}^{3} \bar{b}^{5}\right\rangle, T=\left\langle a^{2} \bar{b}^{2} a^{2} \bar{b}^{5},(a b)^{-3} \bar{b}^{5}\right\rangle$, subgroups of $F(a, b)$, have complexities 17 and 16 respectively (but lengths 21 and 22 repectively). They are equivalent by the Whitehead map $(\{a, b\}, b)$.

\section{REFERENCES}

1. S. M. Gersten, Fixed points of automorphisms of frees groups, Adv. in Math. (to appear). 2. Soc. (N.S.) 8 (1983), 451-454.

3. P. J. Higgins and R. C. Lyndon, Equivalence of elements under automorphisms of a free group, J. London Math. Soc. (2) 8 (1974), 254-258.

4. R. C. Lyndon and P. E. Schupp, Combinatorial group theory, Ergeb. Math. Wiss., Bd. 89, Springer-Verlag, 1977.

5. J. McCool, A presentation for the automorphism group of a free group of finite rank, J. London Math. Soc. (2) 8 (1974), 259-266.

6. __ Some finitely presented subgroups of the automorphism group of a free group, J. Algebra 35 (1975), 205-213.

7. E. S. Rapaport, On free groups and their automorphisms, Acta Math. 99 (1958), 139163.

8. John R. Stallings, Topology of finite graphs, Invent. Math. 71 (1983), 551-565.

9. J. H. C. Whitehead, On certain sets of elements in a free group, Proc. London Math. Soc. 41 (1936), 48-56. 800 .

10. __ On equivalent sets of elements in a free group, Ann. of Math. (2) 37 (1936), 782-

Department of Mathematics, University of Utah, Salt Lake City, Utah 84112 